\title{
Efeitos cardiorrespiratórios e analgésicos da cetamina por via epidural, por infusão intravenosa contínua ou pela associação de ambas, em cães submetidos à osteossíntese de fêmur
}

\author{
Cardiorespiratory and analgesic effects of ketamine via epidural route, intravenous continuous \\ infusion or association of both, in dogs submitted to femoral osteosynthesis
}

\author{
Adriano Bonfim Carregaro ${ }^{\text {I* }}$ Gabrielle Coelho Freitas ${ }^{\text {II }}$ Jenifer de Santana Marques ${ }^{\text {II }}$ \\ Thomas Alexander Trein ${ }^{\text {II }}$ Virgínia Heinze Pohl ${ }^{I I}$ Fabiano Zanini Salbego ${ }^{\text {III }}$ Alceu Gaspar Raiser ${ }^{\text {II }}$
}

\section{RESUMO}

A cetamina tem demonstrado efeito analgésico em doses subanestésicas, além da manutenção da estabilidade dos parâmetros fisiológicos. O estudo objetivou avaliar os efeitos cardiorrespiratórios e a analgesia pós-operatória da cetamina administrada por via epidural, por infusão intravenosa contínua ou pela associação de ambas, em cães submetidos à osteossíntese de fêmur. Foram utilizadas 25 cadelas, hígidas, distribuídas aleatoriamente em quatro grupos: CEP $\left(2 \mathrm{mg} \mathrm{kg}^{-1}\right.$ de cetamina associada à lidocaína $2 \%$ via epidural), CIV (lidocaína $2 \%$ via epidural e $1 \mathrm{mg}^{\mathrm{kg}^{-1}}$ de cetamina IV seguido de infusão contínua IV com $100 \mu \mathrm{g} \mathrm{kg} \mathrm{min}^{-1}$ da mesma), CIVEP ( $2 \mathrm{mg}_{\mathrm{kg}} \mathrm{k}^{-1}$ de cetamina associada à lidocaína $2 \%$ via epidural e $1 \mathrm{mg} \mathrm{kg}^{-1}$ de cetamina IV, seguido de infusão contínua IV com $100 \mu \mathrm{g} \mathrm{k} \mathrm{min}^{-1}$ ) e CON (anestesia epidural com lidocaína 2\%). Avaliaram-se FC, $f, P A S, P A M, P A D, T^{\circ} \mathrm{C}$, tempo de bloqueio motor e analgesia pós-operatória por meio de escala analógica visual. Houve elevação da FC no CIV e diminuição desse parâmetro no CEP. As pressões arteriais mantiveram-se dentro dos valores fisiológicos e não foram observadas diferenças na fe $T^{\circ} \mathrm{C}$. O tempo de duração do bloqueio anestésico foi potencializado nos grupos que receberam cetamina epidural, diferindo significativamente em relação ao controle. O tempo para a analgesia resgate não diferiu entre os grupos. Conclui-se que a administração de cetamina pela via epidural, por infusão contínua intravenosa ou pela associação de ambas promoveu estabilidade cardiorrespiratória no período transcirúrgico, porém não foi capaz de prolongar a duração da analgesia pós-operatória em cães submetidos à osteossíntese de fêmur.

Palavras-chave: dor pós-operatória, analgesia multimodal, cetamina, cães.

\begin{abstract}
Ketamine has demonstrated analgesic effects in subanesthetic doses, besides the maintenance of stability of physiological parameters. The study aimed to evaluate the cardiorespiratory effects and the post operative analgesia of ketamine via epidural route, intravenous continuous infusion or association of both, in dogs submitted to femoral osteosynthesis. Twenty-five healthy bitches were randomly assigned to four groups: CEP (2mg kg $\mathrm{kg}^{-1}$ of ketamine associated with lidocaine $2 \%$ via epidural route), CIV (lidocaine $2 \%$ via epidural route and $1 \mathrm{mg} \mathrm{kg}^{-1}$ of ketamine $I V$, followed by IV continuous infusion of $100 \mu \mathrm{g} \mathrm{kg} \mathrm{min} \mathrm{mi}^{-1}$ of ketamine), CIVEP (epidural anesthesia identical to CEP and ketamine infusion as in CIV) and CON (epidural anesthesia with lidocaine 2\%). $H R, R R, S A P, M A P, D A P$ and $T^{\circ} C$, sensitive blockade time and post operative analgesia measured with visual analog scale were evaluated. There was an increase in HR in CIV and decrease of this parameter in CEP. Arterial pressures kept within physiological values and differences in $R R$ and $T^{\circ} C$ were not observed. The anesthetic blockade time was augmented in the groups which received epidural ketamine, differing significantly in relation to the control. The time for rescue analgesia did not differ between the groups. It can be concluded the administration of ketamine via epidural route, intravenous continuous infusion or the association of both promoted cardiorespiratory stability during the operative period; however, it was not able to extend the duration of post operative analgesia in dogs submitted to femoral osteosynthesis.
\end{abstract}

Key words: post operative pain, multimodal analgesia, ketamine, dogs.

'Faculdade de Zootecnia e Engenharia de Alimentos, Universidade de São Paulo (USP), Av. Duque de Caxias Norte, 225, 13635900, Pirassununga, SP, Brasil. E-mail: carregaro@usp.br.*Autor para correspondência.

"Universidade Federal de Santa Maria (UFSM), Santa Maria, RS, Brasil.

IIIPontifícia Universidade Católica do Rio Grande do Sul (PUCRS), Porto Alegre, RS, Brasil. 


\section{INTRODUÇÃO}

A cetamina tem despertado crescente interesse em razão de suas propriedades analgésicas, principalmente no período pós-operatório, produzindo analgesia quando administrada em doses subanestésicas (WAGNER et al., 2002). Essa ação é imputada ao efeito antagonista nos receptores $\mathrm{N}$-metilD-aspartato (NMDA), na medula espinhal, os quais estão envolvidos na sensibilização e neuroplasticidade do Sistema Nervoso Central (SNC) (WU et al., 2000). Além disso, também atua em receptores opióides e muscarínicos (SEAR, 1996). Quando administrada como parte de um protocolo de anestesia balanceada, permite a redução do requerimento de anestésicos, mantendo os parâmetros hemodinâmicos estáveis (BETTSCHART-WOLFENSBERGER \& LARENZA, 2007).

Em humanos, a analgesia produzida pela cetamina foi avaliada pela administração intravenosa, epidural (WANG et al., 2006), intra-articular (BORNER et al., 2006) e infiltrativa (TAN et al., 2007). A administração de $2 \mathrm{mg} \mathrm{kg}^{-1}$ de cetamina pela via epidural em cães anestesiados com isofluorano foi associada a mínimas alterações hemodinâmicas (MARTIN et al., 1997), além de reduzir a dor por até duas horas nessa espécie (HAMILTON et al., 2005). A administração epidural também reduz a hiperalgesia pós-incisional em cães, sem alterar os parâmetros cardiopulmonares (DUQUE et al., 2004). Ademais, produz efeito analgésico aditivo em regimes multimodais pela via epidural (CHIA et al., 1998), potencializando a ação de outros analgésicos e anestésicos locais (MARTINDALE et al., 2004).

A administração de baixas doses de cetamina pela via intravenosa também parece produzir analgesia. A administração de $100 \mu \mathrm{g} \mathrm{kg} \mathrm{min}{ }^{-1}$ em cães reduziu a concentração alveolar mínima do sevofluorano (WILSON et al., 2008), além de manter estáveis os parâmetros hemodinâmicos de cães hipovolêmicos (HONSHO et al., 2004; DUQUE et al., 2005).

O objetivo deste estudo foi avaliar os efeitos da cetamina pela via epidural, por infusão intravenosa contínua ou pela associação de ambas nos parâmetros cardiorrespiratórios, durante o período transoperatório, e na analgesia pós-operatória em cães submetidos à osteossíntese de fêmur.

\section{MATERIAL E MÉTODOS}

Foram utilizadas 25 cadelas adultas, sem raça definida e com peso de $14 \pm 2,7 \mathrm{~kg}$, as quais foram submetidas à osteossíntese de fêmur (estudo paralelo). Para o procedimento, os animais foram mantidos sob anestesia epidural lombossacral, sendo distribuídos aleatoriamente em quatro grupos. $\mathrm{OCEP}(\mathrm{n}=7)$ recebeu $2 \mathrm{mg} \mathrm{kg}^{-1}$ de cetamina a associada à lidocaína ${ }^{\mathrm{b}} 2 \%$ sem vasoconstritor ( $\mathrm{s} / \mathrm{v})$ via epidural, perfazendo um volume final de $1 \mathrm{~mL}$ para cada $5 \mathrm{~kg}$, e bolus intravenoso de $1 \mathrm{~mL}$ de solução salina seguido de infusão intravenosa contínua de $10 \mathrm{~mL} \mathrm{~kg} \mathrm{~h}^{-1} ; \mathrm{o}$ CIV (n=7) recebeu anestesia epidural com lidocaína $2 \% \mathrm{~s} / \mathrm{v}\left(1 \mathrm{~mL} 5 \mathrm{~kg}^{-1}\right)$ e $1 \mathrm{mg} \mathrm{kg}^{-1}$ de cetamina IV em bolus, seguida de infusão contínua

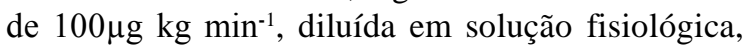
perfazendo um volume final de $10 \mathrm{~mL} \mathrm{~kg} \mathrm{~h}^{-1}$; o CIVEP $(\mathrm{n}=7)$ recebeu $2 \mathrm{mg} \mathrm{kg}^{-1}$ de cetamina associada à lidocaína $2 \%$ via epidural e $1 \mathrm{mg} \mathrm{kg}^{-1}$ de cetamina IV,

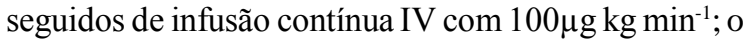
grupo controle $(\mathrm{CON} ; \mathrm{n}=4)$ recebeu anestesia epidural

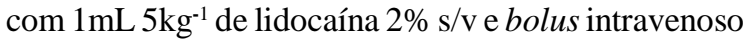
de solução salina $(1 \mathrm{~mL})$ seguido de infusão contínua com solução salina a $10 \mathrm{~mL} \mathrm{~kg} \mathrm{~h}^{-1}$.

Quatro horas antes da cirurgia, os animais foram anestesiados com $4 \mathrm{mg} \mathrm{kg}^{-1}$ de propofol ${ }^{\mathrm{c}}$ para punção da artéria podal dorsal utilizando-se cateter $22 \mathrm{G}$, para mensuração transanestésica das pressões arteriais sistólica (PAS), média (PAM) e diastólica (PAD), em $\mathrm{mmHg}$, por meio de monitor multiparamétrico ${ }^{\mathrm{d}}$, via transdutor de pressão conectado ao cateter arterial. O monitor foi calibrado antes do início das aferições, estabelecendo-se o nível zero na altura do manúbrio. Em seguida, foi aguardada a completa recuperação dos animais.

A medicação pré-anestésica foi realizada com $0,1 \mathrm{mg} \mathrm{kg}^{-1}$ de acepromazina ${ }^{\mathrm{e}} \mathrm{IM}$. Posteriormente, foi realizada venopunção cefálica e colocação de cateter 20G, para posterior infusão. Após 20 minutos, os animais foram induzidos à anestesia com $4 \mathrm{mg} \mathrm{kg}^{-1} \mathrm{de}$ propofol IV, permitindo a punção epidural lombossacral e iniciando-se a infusão contínuaf. O bloqueio epidural foi confirmado pelo relaxamento do esfíncter anal e pela ausência de reflexo interdigital. Quinze minutos após a anestesia epidural, deu-se início ao procedimento cirúrgico. Avaliaram-se a frequência cardíaca $(\mathrm{FC})$, em batimentos por minuto, PAS, PAM, PAD, em $\mathrm{mmHg}$, frequência respiratória $(f)$, em movimentos por minuto e temperatura corporal $\left(\mathrm{T}^{\circ} \mathrm{C}\right)$, em graus Celsius, antes da anestesia epidural ( 0 min) e a cada $10 \mathrm{~min}$, por um observador que desconhecia qual dos tratamentos os animais haviam recebido.

A analgesia pós-operatória foi realizada por dois avaliadores, que também desconheciam os tratamentos administrados aos cães. As avaliações foram realizadas a cada hora até o momento em que fora necessária a administração da analgesia de resgate. 
A avaliação analgésica foi realizada por meio de escala analógica visual (EAV), a qual era atribuída considerando-se o comportamento de cada animal, na gaiola e fora dela. Também foi avaliado o tempo de duração do bloqueio motor por meio de pinçamento interdigital. Caso fossem imputadas notas acima de 50 na EAV, eram administrados $0,5 \mathrm{mg} \mathrm{kg}^{-1}$ de morfina ${ }^{\mathrm{g}} \mathrm{IM}$ e $0,2 \mathrm{mg} \mathrm{kg}^{-1}$ de meloxicam ${ }^{\mathrm{h}} \mathrm{IM}$.

A análise estatística ${ }^{i}$ foi realizada utilizandose análise de variância (ANOVA) para amostras pareadas, com posterior teste de Dunnett para comparações de médias dentro de cada grupo em relação ao 0 min. Para comparações entre os grupos, em cada momento, foi realizada ANOVA, seguida do teste de Tukey. A comparação do tempo de bloqueio e do tempo até o resgate foi realizada por ANOVA, com posterior teste de Dunn. Os valores fisiológicos estão apresentados como médias \pm erros-padrão. Os tempos de duração do bloqueio e a necessidade de analgesia de resgate estão apresentados como medianas e intervalos interquartis. As diferenças foram consideradas significativas quando $\mathrm{P}<0,05$.

\section{RESULTADOS}

A anestesia epidural foi realizada com sucesso em todos os animais, sendo observadas condições adequadas de miorrelaxamento e analgesia durante o procedimento cirúrgico, o qual foi padronizado no tempo de 120 minutos por ser realizado em condições experimentais. Dois animais foram removidos do estudo, um do CIV e um do CIVEP, por apresentarem hemorragia iatrogênica intensa durante o procedimento cirúrgico.

A FC apresentou variação significativa em relação ao 0 min apenas no $\mathrm{CEP}$, observando-se diminuição entre 50 e 100min. Na comparação dos grupos, observou-se aumento da FC, no CIV, quando comparado ao CEP e CON, dos 10 aos 30min e em 60 e 80min (Figura 1). Entretanto, a FC foi mantida em valores fisiológicos aceitáveis para a espécie.

Houve decréscimo da PAS em todos os grupos apenas em 10min. A PAM foi superior no CEP entre 100 e $110 \mathrm{~min}$. Nesse mesmo intervalo, na comparação dos grupos, CIV e CEP apresentaram maiores valores em relação ao CIVEP. A PAD foi superior no CEP entre 70 e 120min. O CIVEP apresentou elevação da PAD em relação ao CON em 40min e redução em relação ao CEP em 100min (Figura 1). Os valores de $f$ e temperatura retal não diferiram em relação ao 0min ou entre os grupos em nenhum dos momentos avaliados, permanecendo entre 15 e $30 \mathrm{mov}^{\mathrm{min}^{-1}}$ e $36 \mathrm{e}$ $38^{\circ} \mathrm{C}$, respectivamente.
O tempo de duração do bloqueio motor foi maior no CIVEP [4 $(3 ; 4)$ horas], sendo diferente do CIV [2 (2;3) horas] e CON [2 (1;3) horas]. O CEP apresentou o segundo maior tempo de bloqueio [3 $(2 ; 5)$ horas], não diferindo de nenhum dos grupos (Figura 2). $\mathrm{O}$ tempo até a administração de analgesia resgate foi maior no CIVEP [4,5 (4;7) horas], mas não diferindo estatisticamente do CEP [ $4(3 ; 5)$ horas], CON [4 (3;5) horas] e CIV [4 (4;5) horas] (Figura 2).

\section{DISCUSSÃO}

Usualmente, a cetamina aumenta o débito cardíaco, a frequência cardíaca e a pressão arterial (JACOBSON \& HARTSFIELD, 1993; BERGMAN, 1999; OKLU et al., 2003), sendo até mesmo recomendada em pacientes com alto risco anestésico e suscetíveis à depressão cardiovascular e hipotensão (DUQUE et al., 2005). Estudos prévios demonstraram que a infusão

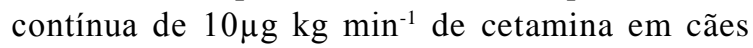
promoveu taquicardia (WHITE et al., 1982; MUIR et al., 2003); porém, a administração de uma dose bolus IV de $3 \mathrm{mg} \mathrm{kg}^{-1}$ não registrou tal efeito (SOLANO et al., 2006). Neste estudo, os grupos diferiram entre si na análise da FC dos 10 aos 30min, nos quais os grupos tratados com cetamina apresentaram discreta elevação, mas permanecendo dentro dos parâmetros fisiológicos, resultado também descrito com o uso da cetamina pela via epidural (DUQUE et al., 2004).

A taquicardia observada com o uso de cetamina deve-se à estimulação direta do SNC, com aumento do tônus simpático, sendo o principal mecanismo responsável pela estimulação cardiovascular relatado em humanos (STOELTING \& HILLIER, 2005). Em razão da ação da cetamina nos receptores opióides, especialmente em baixas doses, são observados efeitos analgésicos mais significativos do que os efeitos estimulatórios do sistema nervoso simpático frequentemente observados (HORN \& NESBIT, 2004; BETTSCHART-WOLFENSBERGER \& LARENZA, 2007).

Neste estudo, os animais do CEP apresentaram diminuição significativa da FC em relação ao basal após 50min. Deve ser considerado que a administração epidural de lidocaína pode promover bradicardia (IFF \& MOENS, 2008), em decorrência do bloqueio simpático (HONG, 2008). A estimulação simpática produzida pela cetamina pode ter antagonizado o bloqueio simpático produzido pela lidocaína nos grupos que receberam cetamina IV, mas não no CEP e CON, que apresentaram valores inferiores de FC. 


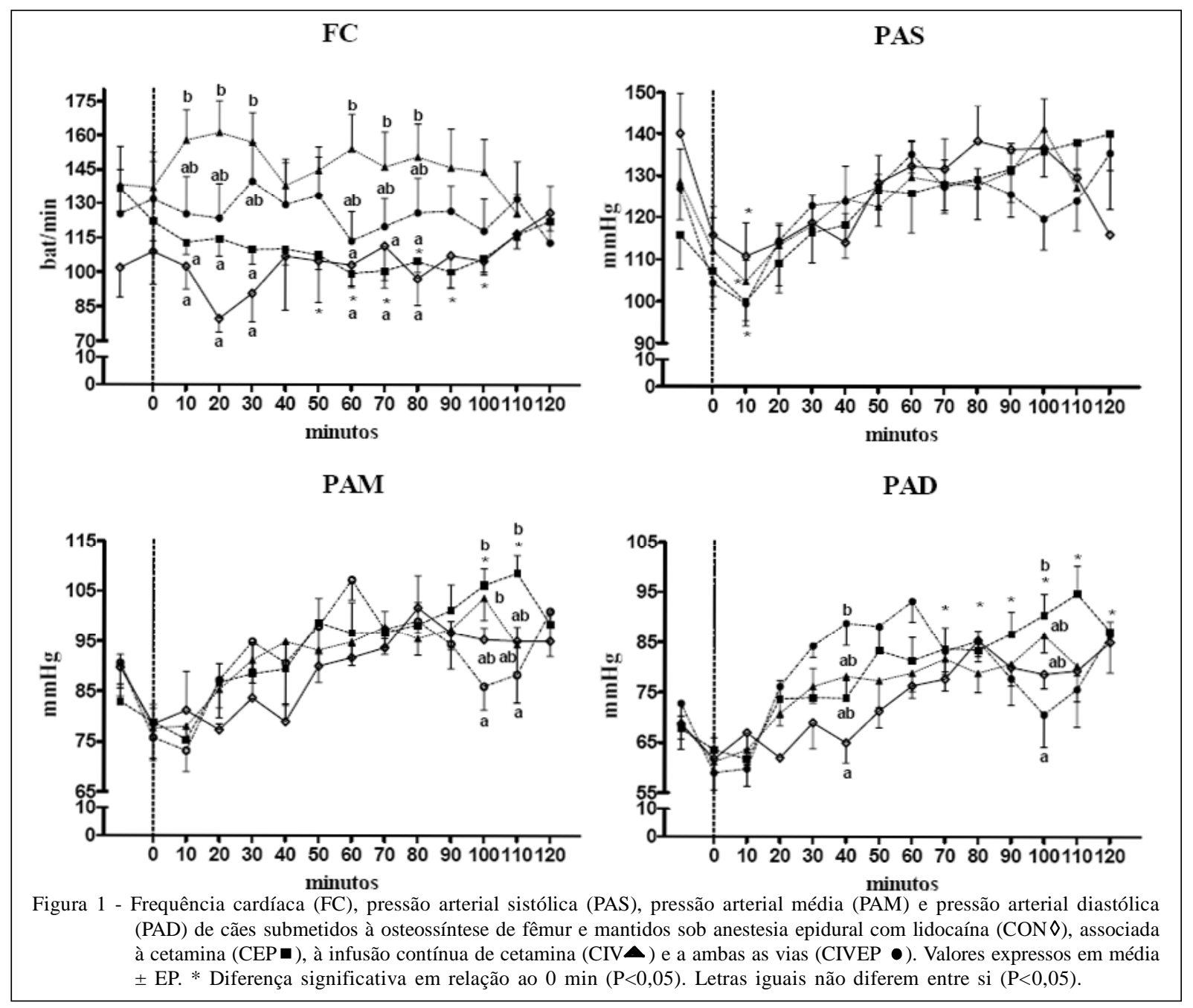

As pressões arteriais mantiveram-se estáveis e dentro dos valores fisiológicos durante as avaliações. Sugere-se que a queda da PAS observada aos 10min de avaliação pode ser imputada à administração de propofol para posterior realização de punção epidural. Em cães, a administração epidural de cetamina não promoveu alterações na pressão arterial (MARTIN et al., 1997), assim como o seu uso por infusão intravenosa contínua de $100 \mu \mathrm{g} \mathrm{kg} \mathrm{min}^{-1}$, mesmo em cães hipovolêmicos (HONSHO et al., 2004). Ademais, cães tratados com cetamina não sofreram alterações na ventilação, mas sabe-se que a depressão respiratória está associada a doses elevadas ou à interação com outros depressores do SNC, resultando em hipercapnia e hipóxia (JACOBSON \& HARTSFIELD, 1993).

O tempo de duração do bloqueio motor foi maior nos grupos que receberam cetamina por via epidural. Isso ocorreu em razão das propriedades anestésicas locais do fármaco e da sua interação com canais de sódio, resultando em bloqueio motor, que em cães tem duração de aproximadamente 30 minutos com o uso de $2 \mathrm{mg} \mathrm{kg}^{-1}$ (DUQUE et al., 2004). Esse efeito parece ser dose-dependente, visto que a administração epidural de doses menores de cetamina não produziu bloqueio motor em cães (VALADÃO et al., 2002). Dessa forma, sugere-se que houve sinergismo da cetamina à lidocaína, prolongando o período de bloqueio motor.

A analgesia produzida pela cetamina administrada tanto pela via epidural, quanto por infusão intravenosa contínua já foi relatada em humanos e animais. A administração epidural de cetamina produziu analgesia no período pós-operatório imediato em ovelhas (GUEDES et al., 2006), promoveu analgesia de curto prazo em pessoas (WILSON et al., 2008) e reduziu a pontuação de dor por duas horas em cães (HAMILTON et al., 2005). No entanto, o presente estudo demonstrou que a administração preventiva da cetamina por via epidural, por infusão contínua intravenosa ou pela associação de ambas não foi capaz de prolongar significativamente a duração da analgesia pós-operatória. 


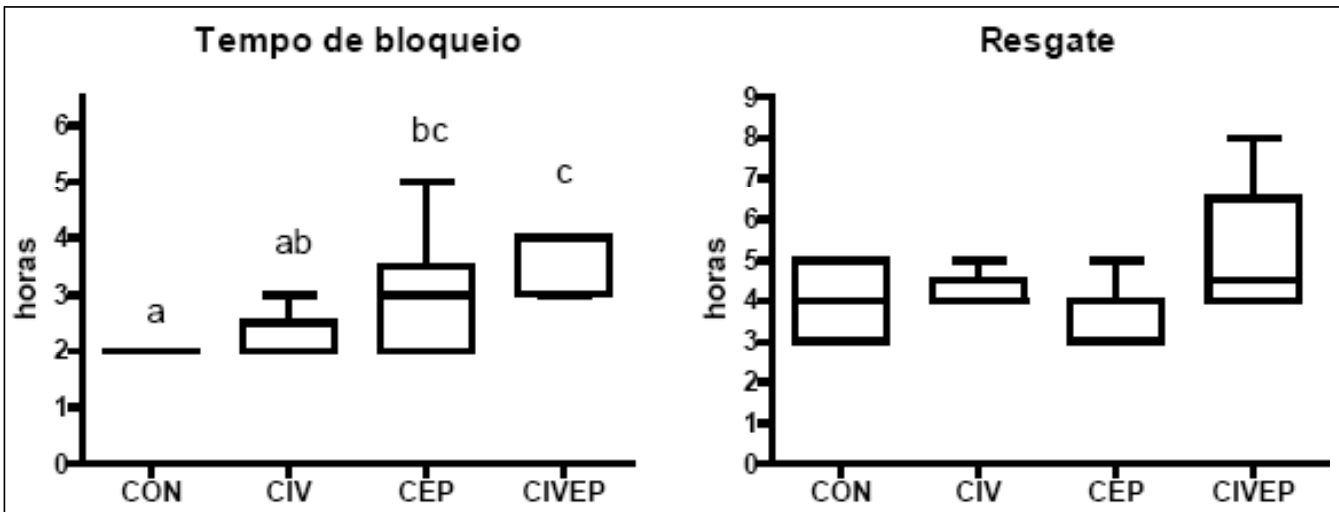

Figura 2 - Tempo de duração do bloqueio motor e tempo até administração de analgesia resgate no período pósoperatório de cães submetidos à osteossíntese de fêmur e mantidos sob anestesia epidural com lidocaína $(\mathrm{CON})$, associada à cetamina (CEP), à infusão contínua de cetamina (CIV) e a ambas as vias (CIVEP). Valores expressos em mediana e intervalo interquartil. Letras iguais não diferem entre si $(\mathrm{P}<0,05)$.

Cabe ressaltar que o procedimento cirúrgico empregado neste estudo é considerado de dor intensa, e sugere-se que o protocolo anestésico com o uso de cetamina por diferentes vias seja benéfico em procedimentos menos traumáticos. Além disso, modelos cirúrgicos com dor moderada a intensa requerem a associação de opióides no protocolo de analgesia pósoperatória (MENINGAUX et al., 2000), reduzindo-se assim a dose utilizada. WAGNER et al. (2002)

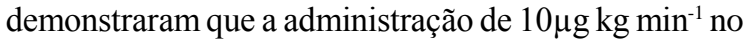
período pré-operatório melhorou a analgesia e o conforto pós-cirúrgico de cães submetidos à amputação. Porém, esse estudo utilizou um protocolo que incluiu opióides. Com o objetivo de avaliar a cetamina como único fármaco, optou-se, no presente estudo, pela não utilização de outros analgésicos, bem como pelo uso de uma dose mais elevada.

O grupo CIVEP, apesar de não ter diferido estatisticamente dos outros grupos, clinicamente apresentou o maior tempo até administração da analgesia de resgate. Este resultado pode ser atribuído ao sinergismo entre as vias epidural e intravenosa, ou à maior dose total de cetamina administrada aos animais deste grupo. Existem evidências de efeito dosedependente da cetamina na redução do consumo pósoperatório de morfina (CHERRY et al., 1995). Além disso, a administração preventiva epidural de cetamina reduz os mecanismos de hiperalgesia secundária (HARTRICK et al., 1997).

\section{CONCLUSÃO}

Conclui-se que a administração de cetamina pela via epidural, por infusão contínua intravenosa ou pela associação de ambas promoveu estabilidade cardiorrespiratória no período transcirúrgico. A cetamina epidural prolongou a duração do bloqueio produzido pela lidocaína. A administração de cetamina pela via epidural, por infusão contínua intravenosa ou pela associação de ambas não foi capaz de prolongar a duração da analgesia pós-operatória em cães submetidos à osteossíntese de fêmur, não sendo recomendada como único analgésico nesse procedimento cirúrgico.

\section{COMITÊ DE ÉTICA E BEM ESTAR ANIMAL}

Este trabalho foi aprovado pelo Comitê de Ética e Bem-estar Animal da Instituição de origem, sob o protocolo de número 30/2008, por estar de acordo com os princípios éticos do Colégio Brasileiro de Experimentação Animal.

\section{FONTES DE AQUISIÇÃO}

a- Ketalar ${ }^{\circledR}$, Parke-Davis, Rio de Ja

neiro, RJ, Brasil.

b- Xylestesin ${ }^{\circledR}$, Astra Química e Farmacêutica, São Paulo, SP, Brasil.

c- Propofol ${ }^{\circledR}$, Cristália Prod. Quím. Farm. LTDA, Itapira, SP, Brasil.

d- PM-9000 Express - Mindray® Medical Intenational Ltd, Shenzhen, China.

e- Acepran ${ }^{\circledR}$ 0,2\%, UNIVET S.A, São Paulo, SP, Brasil.

f- Bomba de infusão por seringa B. Braun CC, modelo 8714827, Laboratórios B. Braun. S.A., São Gonçalo, RJ, Brasil.

g- Dimorf ${ }^{\circledR}$, Cristália Prod. Quím.Farm. LTDA, Itapira, SP, Brasil.

h- Maxicam ${ }^{\circledR} 2 \%$, Ouro Fino Saúde Animal, Cravinhos, SP, Brasil.

i- GraphPad Prism, GraphPad Software Inc, San Diego, California, USA. 


\section{REFERÊNCIAS}

BERGMAN, S.A. Ketamine: review of its pharmacology and its use in pediatric anesthesia. Anesthesia Progress, v.46, p.10-20, 1999. Disponível em: <http://www.ncbi.nlm.nih.gov/ pmc/articles/PMC2148883/?page=1>. Acesso em: 13 jun. 2010.

BETTSCHART-WOLFENSBERGER, R.; LARENZA, M.P. Balanced anesthesia in the equine. Clinical Techniques in Equine Practice, v.6, p.104-110, 2007. Disponível em: <http://www.sciencedirect.com/ science?_ob=ArticleURL\&_udi=B7GW6-4PFCGHK$4 \&$ _user $=687358 \&$ _coverDate $=06 \% 2 \mathrm{~F} 30 \% 2 \mathrm{~F} 2007 \&$ _ rdoc $=1 \&$ fmt $=$ high \&_orig $=$ search\&_sort $=$ d\&_docanchor $=\&$ view $=$ c\&_search StrId $=1368481987 \&$ _rerunOrigin $=$ go ogle\&_acct $=$ C $000037899 \&$ \&ersion $=1 \&$ \&urlVersio $\mathrm{n}=0$ \&_userid=687358\&md5=f33e60e37e06f41a8d2ba998dc2d2044>. Acesso em: 13 jun. 2010. doi: 10.1053/j.ctep.2007.05.002.

BORNER, M. et al. Intra-articular ketamine after arthroscopic knee surgery: optimisation of postoperative analgesia. Anaesthesia, v.18, n.8, p.563-569, 2006. Disponível em: <http://www.springerlink.com/content/71524154341113p0/ >. Acesso em: 13 jun. 2010. doi: 10.1007/s00101-007-1249-y.

CHERRY, D.A. et al. Ketamine as an adjunct to morphine in the treatment of pain. Pain, v.62, p.119-121, 1995. Disponível em: <http:/ /www.sciencedirect.com/science?_ob=MImg\&_imagekey=B6T0K3 YYV2JG-1W-1\&_cdi $=4865 \&$ _user $=687358 \&$ \&ii $=03043$ $9599500010 \mathrm{P} \&$ _orig = search \&_coverDate $=0$ 7\%2F31\%2F1995\&_sk=999379998\&view $=\mathrm{c} \& \mathrm{wchp}=\mathrm{dGLbVtz}-$ zSkWA\&md5=7ec4f95f7e575e902923008f820567ea\&ie $=$ / sdarticle.pdf>. Acesso em: 13 jun. 2010. doi: 10.1016/03043959(95)00010-P.

CHIA, Y.Y. et al. Adding ketamine in a multimodal patientcontrolled epidural regimen reduces postoperative pain and analgesic consumption. Anesthesia and Analgesia, v.86, n.6, p.1245-1249, 1998. Disponível em: <http:// www.anesthesia-analgesia.org/content/86/6/1245.long >. Acesso em: 13 jun. 2010.

DUQUE, M.J.C. et al. Pre-emptive epidural Ketamine or S(+)Ketamine in post-incisional pain in dogs: a comparative study. Veterinary Surgery, v.33, p.361-367, 2004. Disponível em: <http://www3.interscience.wiley.com/cgi-bin/fulltext/ 118771331/PDFSTART>. Acesso em: 13 jun. 2010. doi:10.1111/j.1532-950X.2004.04052.x.

DUQUE, M.J.C. et al. Continuous infusion of ketamine in hypovolemic dogs anesthetized with desflurane. Journal of the Veterinary Emergency and Critical Care, v.15, n.2, p.9299, 2005. Disponível em: <http://www3.interscience.wiley.com/ cgi-bin/fulltext/118715845/PDFSTART>. Acesso em: 13 jun. 2010. doi: 10.1111/j.1476-4431.2005.00133.x.

JACOBSON, J.D.; HARTSFIELD, S.M. Cardiovascular effects of intravenous bolus administration and infusion of ketaminemidazolam in isoflurane-anesthetized dogs. American Journal of the Veterinary Research, v.54, p.1715-1720, 1993.

GUEDES, A.G. et al. Effects of preoperative epidural administration of racemic ketamine for analgesia in sheep undergoing surgery. American Journal of the Veterinary Research, v.67, n.2, p.222-229, 2006. doi: 10.2460/ ajvr.67.2.222.
HAMILTON, S.M. et al. Evaluation of analgesia provided by the administration of epidural ketamine in dogs with a chemically induced synovitis. Veterinary Anaesthesia and Analgesia, v.32, p.30-39, 2005. Disponível em: <http:// www3.interscience.wiley.com/cgi-bin/fulltext/118670351/ PDFSTART>. Acesso em: 13 jun. 2010. doi: 10.1111/j.14672995.2004.00171.x.

HARTRICK, C.P. et al. Preemptive intrathecal ketamine delays mechanical hiperalgesia in the neuropathic rat. Anesthesia and Analgesia, v.86, p.557-560, 1997. Disponível em: <http:/ /www.anesthesia-analgesia.org/content/86/3/557.long>. Acesso em: 13 jun. 2010.

HONG, J.Y. Haemodynamic and ventilatory effects of preoperative epidural analgesia during laparoscopic hysterectomy using $\mathrm{NICO}^{\mathrm{TM}}$. Singapore Medical Journal, v.49, n.3, p.233-238, 2008. Disponível em: <http:// smj.sma.org.sg/4903/4903a9.pdf>. Acesso em: 13 jun. 2010.

HONSHO, C.S. et al. Efeitos da infusão contínua de cetamina sobre a pressão intra-ocular em cães hipovolêmicos anestesiados com desflurano. Arquivo Brasileiro de Medicina Veterinaria e Zootecnia, v.56, n.5, p.610-617, 2004 Disponível em: <http://www.scielo.br/scielo.php?pid=S0102$09352004000500007 \&$ script=sci_arttext $>$. Acesso em: 13 jun. 2010. doi: 10.1590/S0102-09352004000500007.

HORN, E.; NESBIT, S.A. Pharmacology and pharmacokinetics of sedatives and analgesics. Gastrointestinal Endoscopy Clinics of North America, v.14, p.247-268, 2004. Disponível em: <http:/ /www.sciencedirect.com/science? ob=ArticleURL\& udi=B7RM14FK168W-67\&_user $=687358 \&$ \&coverDate $=04 \% 2 \mathrm{~F} 30 \% 2 \mathrm{~F} 2004 \&$ _rdoc $=1 \&$ \& fmt $=$ high\&_orig $=$ search\&_sort $=$ d\&_docanchor $=\&$ view $=$ c\&_acct $=$ C $000037899 \&$ \& version $=1 \&$. u r 1 Vers i o $n=0 \&$ u s e ri d $=687358 \&$ m d 5 $=a 37 \mathrm{a} 26 \mathrm{fd} 783 \mathrm{~b} 7 \mathrm{~d} 52 \mathrm{fbd} 8607 \mathrm{ea} 67 \mathrm{~d} 07 \mathrm{~d} 7>$. Acesso em: 12 jun. 2010. doi: 10.1016/j.giec.2004.01.001.

IFF, I.; MOENS, Y. Two cases of bradyarrhythmia and hypotension after extradural injection in dogs. Veterinary Anesthesia and Analgesia, v.35, p.265-269, 2008. Disponível em: <http://www3.interscience.wiley.com/cgi-bin/ fulltext/119421077/HTMLSTART>. Acesso em: 13 jun. 2010. doi: $10.1111 /$ j.1467-2987.2007.00373.x.

MARTIN, D.D. et al. Hemodynamic effects of epidural ketamine in isoflurane-anesthetized dogs. Veterinary Surgery, v.26, n.6, p.505-509, 1997.

MARTINDALE, S.J. et al. Double-blind randomized controlled trial of caudal versus intravenous $\mathrm{S}(+)$-ketamine for supplementation of caudal analgesia in children. British Journal of Anaesthesia, v.92, n.3, p.344-347, 2004. Disponível em: <http://bja.oxfordjournals.org/cgi/content/full/ 92/3/344 ?view=long \&pmid=14742331 >. Acesso em: 13 jun. 2010. doi: 10.1093/bja/aeh076.

MENINGAUX, C. et al. The benefits of intraoperative smalldose ketamine on postoperative pain after anterior cruciate legament repair. Anesthesia and Analgesia, v.90, p.129135, 2000. Disponível em: <http://www.anesthesia-analgesia.org/ content/90/1/129.long>. Acesso em: 13 jun. 2010.

MUIR, W.W. et al. Effects of morphine, lidocaine, ketamine and morphine-lidocaine-ketamine drug combination on 
minimum alveolar concentration in dogs anesthetized with isoflurane. American Journal of the Veterinary Research, v.64, n.9, p.1155-1160, 2003. doi: 10.2460/ajvr.2003.64.1155.

OKLU, E. et al. Which anesthetic agent alters the hemodynamic status during pediatric catheterization? Comparison of propofol versus ketamine. Journal of Cardiothoracic and Vascular Anesthesia, v.17p. 686-690, 2003. Disponível em: <http:// www.sciencedirect.com/science?_ob=ArticleURL\&_udi=B6WHH4B 7PCFP-7\&_user $=687358 \&$ \&coverDate $=12 \% 2 \mathrm{~F} 31 \% 2 \mathrm{~F}$ $2003 \&$ rdoc $=1 \&$ \& f m t $=$ high \&_orig $=\mathrm{search}$ $\&_{-}$s o r t $=$d \&_d o c a n c h o r $=\&$ v i e w $=$ c \& $\&_{-}$a cct $=$ C000037899\&_version $=1 \&$ u rlVersion $=0$ \&_userid $=687358$ $\& m d 5=a 295 d a 0 f 7 c 181 f 27 a 2 a 286 e c 0 b f b b c b b>$. Acesso em: 13 jun. 2010. doi: $10.1053 /$ j.jvca.2003.09.009

SEAR, J.W. Intravenous hypnotics: propofol, ketamine and eltanolone. Current Opinion in Anesthesiology, v.9, p.289-294, 1996.

SOLANO, A.M. et al. Effect of intravenous administration of ketamine on the minimum alveolar concentration of isoflurane in anesthetized dogs. American Journal of the Veterinary Research, v.67, n.1, p.21-25, 2006. doi: 10.2460/ajvr.67.1.21.

STOELTING, R.K.; HILLIER, S.C. In: Pharmacology and physiology in anesthetic practice. 2.ed. Philadelphia: Lippincott Williams \& Wilkins, 2005. Cap.6, p.151-178

TAN, P.H. et al. Preincisional subcutaneous infiltration of ketamine supresses postoperative pain after circumcision surgery. Clinical Journal of Pain, v.23, p.214-218, 2007. Disponível em: <http:// o v i d s p.t x . o v i d . c o m/s p - 2.3.1 b/ ovidweb.cgi?QS2 $=434 \mathrm{f} 4 \mathrm{e} 1 \mathrm{a} 73 \mathrm{~d} 37 \mathrm{e} 8 \mathrm{c} 8 \mathrm{~b} 3 \mathrm{eab} 7 \mathrm{e} 2 \mathrm{fc} 8 \mathrm{fd} 7 \mathrm{c} 8 \mathrm{ffb} 13 \mathrm{ad} 51$ $72 \mathrm{bb} 23 \mathrm{~d} 50 \mathrm{cb} 0483411767736 \mathrm{fa} 56971$ a $2777 \mathrm{f} 8 \mathrm{~d} 09$ 4e7f9231fbc2ab07dc7f3e4fef9841b41496b9ffe1865d8cf846e343354 $4 d 8 d f 7 c 4897153 a f 74 a 50 a b c 5 d f 7 d 11566 c a 1$ fc $5 d 689713 \mathrm{e} 43 \mathrm{~d}$ bbdf 52 bc 3 ad 714 d 6979 cdc 3663 e 2 ce 1 ef 0 a 7 ed 79 17714 b 939 a 490 e 19 e 6 e 3474 c 129 fd 9 e 6 cf 64 5 b a 1 a 925 e 517 a 412026380 b 2 d fe 5290 a 2 d 3 b $71 \mathrm{~d} 08$ a 1 d $807 \mathrm{cfa} 773 \mathrm{fca} 8 \mathrm{f} 5 \mathrm{ddd} 5 \mathrm{~b} 14 \mathrm{c} 76$ 7 a $3 \mathrm{f} 7 \mathrm{df} 472501408920520 \mathrm{a} 4 \mathrm{~d} 4 \mathrm{e} 93 \mathrm{a} 660 \mathrm{~b} 970 \mathrm{~d}$ a 4125869 c b 9093 b 2 fd 0358 bc 8 ac 3072 f 4 d 688 afb3f3e6a62994d7c746e47eee227ad5761e30f62a66387046>. Acesso em: 13 jun. 2010. doi: 10.1097/AJP.0b013e31802e3377.

VALADÃO, C.A.A. et al. Avaliação dos efeitos analgésicos da morfina ou cetamina em cães, após injeção epidural, através dos filamentos de von Frey. Arquivo Brasileiro de Medicina Veterinaria e Zootecnia, v.54, p.383-389, 2002. Disponível em:
$<$ http://www.scielo.br/scielo.php?script=sci_arttext\&pid=S010209352002000400009>. Acesso em: 13 jun. 2010. doi: 10.1590/ S0102-09352002000400009.

WAGNER, A.E. et al. Use of low doses of ketamine administered by constant rate infusion as an adjunct for postoperative analgesia in dogs. Journal of the American Veterinary Medical Association, v.221, n.1, p.72-75, 2002. doi: 10.2460/javma.2002.221.72.

WANG X. et al. Improved postoperative analgesia with coadministration of epidural ketamine and midazolam. Journal of Clinical Anesthesia, v.18, p.563-569, 2006. Disponível em: <http://www.sciencedirect.com/ science?_ob=ArticleURL\&_udi=B6T83-4MK6WM6$3 \&$ user $=687358 \&$ \&coverDate $=12 \% 2 \mathrm{~F} 31 \% 2 \mathrm{~F} 2006 \&$ \&doc $=$ $1 \&$ fmt $=$ high\&_orig $=$ search\&_sort $=$ d\&_docancho $\mathrm{r}=\& \mathrm{vie} w=\mathrm{c}_{-} \mathrm{acct}_{\mathrm{c}} \mathrm{C} 000037899 \&$ \& versio $\mathrm{n}=1 \&$ \& ur l Version $=0 \&$ \& u se rid $=687358 \&$ md5=3c6e2624de467d120c5f0322c0628c43>. Acesso em: 13 jun. 2010. doi: 10.1016/j.jclinane.2006.03.023.

WHITE, P.F. et al. Ketamine - its pharmacology and therapeutic uses. Anesthesiology, n.56, p.119-136, 1982.

WILSON, J. et al. Effects of intravenous lidocaine, ketamine, and the combination on the minimum alveolar concentration of sevoflurane in dogs. Veterinary Anaesthesia and Analgesia, v.35, p.289-296, 2008. Disponível em: <http:// www3.interscience.wiley.com/journal/120089051/abstract>. Acesso em: 13 jun. 2010. doi: 10.1111/j.14672995.2007.00389.x.

WILSON, J.A. et al. A randomized double blind trial of the effect of preemptive epidural ketamine on persistent pain after lower limb amputation. Pain, v.135, p.108-118, 2008. Disponível em: <http:/ /www.sciencedirect.com/science?_ob=ArticleURL\&_udi=B6T0K4P127F6-4\&_user $=687358 \&$ _coverDate $=03 \% 2 \mathrm{~F} 31 \% 2 \mathrm{~F} 20$ $08 \&$ rdoc $=1 \&$ f $\mathrm{mt}=\mathrm{high} \&$ _orig $=$ search\&_sort $=\mathrm{d} \&$ \&do c anchor $=\& v i e w=c \&$ a c ct $=$ C $000037899 \&$ versi o $n=1 \&$ \& ur 1 Version $=0 \&$ \& userid $=687358 \&$ md5=87a5b3ab220f2670e499b68d274c97fc >. Acesso em: 13 jun. 2010. doi: 10.1016/j.pain.2007.05.011

WU, C.T. et al. Pré-incisional epidural ketamine, morphine and bupivacaine combined with epidural and general anesthesia provides pré-emptive analgesia for upper abdominal surgery. Acta Anaesthesiologica Scandinavica, v.44, p.63-68, 2000. Disponível em: <http://www3.interscience.wiley.com/cgi-bin/ fulltext/119179614/HTMLSTART>. Acesso em: 13 jun. 2010. doi: 10.1034/j.1399-6576.2000.440112.x. 